\title{
Phenomenology of gluon TMDs at NNLL
}

\section{Miguel G. Echevarria*}

Departament d'Estructura i Constituents de la Matèria, Universitat de Barcelona, Martí i Franquès 1, 08028 Barcelona, Spain

E-mail: mgechevarriadecm.ub.edu

\begin{abstract}
All the leading-twist (un)polarized gluon transverse momentum dependent parton distribution functions have the same evolution, once they are properly defined in order to cancel spurious rapidity divergences. Currently known perturbative ingredients can be used to resum large logarithms up to next-to-next-to-leading-logarithmic accuracy, putting the theoretical uncertainties under control. This allows a more accurate handling of current and future experimental data, in order to extract valuable information on the role played by gluons in hadron structure.
\end{abstract}

QCD Evolution 2015 -QCDEV2015-

26-30 may 2015

Jefferson Lab (JLAB), Newport News Virginia, USA

${ }^{*}$ Speaker. 


\section{Introduction}

The "naive" definition of transverse momentum dependent distributions (TMDs) introduced in $[1,2]$ and considered in subsequent works, suffers from undesired uncancelled spurious rapidity divergences. The definition of quark TMDs have been recently revisited and updated by Collins [3] and Echevarria-Idilbi-Scimemi [4, 5] (at two loops in Refs. [6, 7]). And it is the goal of this contribution to present the extension to gluon TMDs performed in Ref. [8], relevant for instance in processes such as Higgs boson and quarkonium production in hadron-hadron collisions.

To do so, the Higgs $q_{T}$-distribution in hadron-hadron collisions is considered, with general polarizations, in order to obtain the proper definition for all the leading-twist (un)polarized gluon TMDPDFs. This is crucial to be able to address different processes where they are relevant and perform consistent phenomenological analyses. The cancellation of rapidity divergences is explicitly shown at next-to-leading order (NLO) for the gluon helicity TMDPDF, which represents the distribution of longitudinally polarized gluons inside a longitudinally polarized hadron.

The evolution of the gluon TMDPDFs, as in the case of quark TMDs [9], turns out to be universal, i.e., the same evolution kernel describes the evolution of any of the leading-twist (un)polarized gluon TMDPDFs. This finding is used to quantify the role of linearly polarized gluons inside unpolarized hadrons (see, e.g., [10, 11, 12, 13, 14]), extending the efforts in Ref. [15] by implementing the resummation of large logarithms at next-to-next-to-leading-logarithmic (NNLL) accuracy, providing more accurate predictions and discussing their uncertainty.

\section{Higgs boson $q_{T}$-spectrum and definition of gluon TMDPDFs}

Below we sketch the derivation of the factorization theorem for the Higgs $q_{T}$-distribution in polarized hadron-hadron collisions, $A\left(P, S_{A}\right)+B\left(\bar{P}, S_{B}\right) \rightarrow H\left(m_{H}, q_{T}\right)+X$, by performing a set of consecutive matchings between different effective field theories, relevant at each scale:

$$
\mathrm{QCD}\left(n_{f}=6\right) \rightarrow \mathrm{QCD}\left(n_{f}=5\right) \rightarrow \operatorname{SCET}_{q_{T}} \rightarrow \operatorname{SCET}_{\Lambda_{\mathrm{QCD}}}
$$

More details can be found in Ref. [8]. In the first step we integrate out the top quark mass, $m_{t}$, to build an effective $g g H$ coupling. In the second matching we integrate out the mass of the Higgs boson, $m_{H}$, and obtain a factorized cross-section in terms of well-defined gluon TMDPDFs, which holds for $q_{T} \ll m_{H}$. Those gluon TMDPDFs will be expressed in terms of fundamental hadronic matrix elements. Finally, in the region $\Lambda_{\mathrm{QCD}} \ll q_{T} \ll m_{H}$, we can further refactorize the gluon TMDPDFs in terms of the collinear gluon/quark PDFs, integrating out the large scale $q_{T}$.

After performing the first two matchings, the cross-section can be written as

$$
\begin{aligned}
\frac{d \sigma}{d y d^{2} q_{\perp}} & =2 \sigma_{0}(\mu) C_{t}^{2}\left(m_{t}^{2}, \mu\right) H\left(m_{H}, \mu\right)(2 \pi)^{2} \int d^{2} k_{n \perp} d^{2} k_{\bar{n} \perp} d^{2} k_{s \perp} \delta^{(2)}\left(q_{\perp}-k_{n \perp}-k_{\bar{n} \perp}-k_{s \perp}\right) \\
& \times J_{n}^{(0) \mu v}\left(x_{A}, k_{n \perp}, S_{A} ; \mu\right) J_{\bar{n} \mu v}^{(0)}\left(x_{B}, k_{\bar{n} \perp}, S_{B} ; \mu\right) S\left(k_{s \perp} ; \mu\right)+\mathscr{O}\left(q_{T} / m_{H}\right)
\end{aligned}
$$

where $C_{t}^{2}\left(m_{t}^{2}, \mu\right)$ and $H\left(m_{H}^{2}, \mu\right)$ are the Wilson coefficients that integrate out the top quark and Higgs masses, respectively. $x_{A, B}=\sqrt{\tau} e^{ \pm y}, \tau=\left(m_{H}^{2}+q_{T}^{2}\right) / s$ and $y$ is the rapidity of the produced 
Higgs boson. The Born-level cross section is $\sigma_{0}(\mu)=m_{H}^{2} \alpha_{s}^{2}(\mu) /\left(72 \pi\left(N_{c}^{2}-1\right) s v^{2}\right)$. The pure collinear matrix elements and the soft function are defined as

$$
\begin{aligned}
J_{n}^{(0) \mu v}\left(x_{A}, k_{n \perp}, S_{A} ; \mu\right) & =\frac{x_{A} P^{+}}{2} \int \frac{d y^{-} d^{2} y_{\perp}}{(2 \pi)^{3}} e^{-i\left(\frac{1}{2} x_{A} y^{-} P^{+}-y_{\perp} \cdot k_{n \perp}\right)} \\
& \times \sum_{X_{n}}\left\langle P S_{A}\left|B_{n \perp}^{\mu, a}\left(y^{-}, y_{\perp}\right)\right| X_{n}\right\rangle\left\langle X_{n}\left|B_{n \perp}^{v, a}(0)\right| P S_{A}\right\rangle, \\
J_{\bar{n}}^{(0) \mu v}\left(x_{B}, k_{\bar{n} \perp}, S_{B} ; \mu\right) & =\frac{x_{B} \bar{P}^{-}}{2} \int \frac{d y^{+} d^{2} y_{\perp}}{(2 \pi)^{3}} e^{-i\left(\frac{1}{2} x_{B} y^{+} \bar{P}^{-}-y_{\perp} \cdot k_{\bar{n} \perp}\right)} \\
& \times \sum_{X_{\bar{n}}}\left\langle\bar{P} S_{B}\left|B_{\bar{n} \perp}^{\mu, a}\left(y^{+}, y_{\perp}\right)\right| X_{\bar{n}}\right\rangle\left\langle X_{\bar{n}}\left|B_{\bar{n} \perp}^{v, a}(0)\right| \bar{P} S_{B}\right\rangle, \\
S\left(k_{S \perp} ; \mu\right) & =\frac{1}{N_{c}^{2}-1} \sum_{X_{s}} \int \frac{d^{2} y_{\perp}}{(2 \pi)^{2}} e^{i y_{\perp} \cdot k_{s \perp}}\left\langle 0\left|\left(\mathscr{S}_{n}^{\dagger} \mathscr{S}_{\bar{n}}\right)^{a b}\left(y_{\perp}\right)\right| X_{S}\right\rangle\left\langle X_{S}\left|\left(\mathscr{S}_{\bar{n}}^{\dagger} \mathscr{S}_{n}\right)^{b a}(0)\right| 0\right\rangle .
\end{aligned}
$$

In Ref. [8] one can find the particular definitions of the collinear and soft Wilson lines and the SCET fields.

As shown explicitly at NLO in Ref. [8], for unpolarized, linearly polarized and helicity gluon TMDPDFs, the collinear and soft matrix elements defined above are individually ill-defined, since they contain spurious rapidity divergences. Then, based on Refs. $[4,5,9,3]$, and using $\eta_{n(\bar{n})}$ to label generic parameters that regulate the rapidity divergences present in the (anti-)collinear and soft matrix elements, we define the TMDPDFs as

$$
\begin{aligned}
& \tilde{G}_{g / A}^{\mu v}\left(x_{A}, b_{\perp}, S_{A} ; \zeta_{A}, \mu\right)=\tilde{J}_{n}^{(0) \mu v}\left(x_{A}, b_{\perp}, S_{A} ; \mu ; \eta_{n}\right) \tilde{S}_{-}\left(b_{T} ; \mu ; \eta_{n}\right), \\
& \tilde{G}_{g / B}^{\mu v}\left(x_{B}, b_{\perp}, S_{B} ; \zeta_{B}, \mu\right)=\tilde{J}_{\bar{n}}^{(0) \mu v}\left(x_{B}, b_{\perp}, S_{B} ; \mu ; \eta_{\bar{n}}\right) \tilde{S}_{+}\left(b_{T} ; \mu ; \eta_{\bar{n}}\right) .
\end{aligned}
$$

Here $\zeta_{A, B}$ represent auxiliary rapidity scales, the twiddle labels the functions in coordinate space and we have split the soft function in rapidity space as [5]

$$
\tilde{S}\left(b_{T} ; \mu ; \eta_{n}, \eta_{\bar{n}}\right)=\tilde{S}_{-}\left(b_{T} ; \mu ; \eta_{n}\right) \tilde{S}_{+}\left(b_{T} ; \mu ; \eta_{\bar{n}}\right) \text {. }
$$

The arbitrariness in the choice of the rapidity cutoff to split the soft function, which is not explicitly shown in the above equation, manifests itself as the appearance of the auxiliary energy scales $\zeta_{A}$ and $\zeta_{B}$, which are bound together by $\zeta_{A} \zeta_{B}=q^{4}=m_{H}^{4}$. With properly defined gluon TMDPDFs, the cross-section for the Higgs $q_{T}$-distribution is now given by:

$$
\begin{aligned}
\frac{d \sigma}{d y d^{2} q_{\perp}} & =2 \sigma_{0}(\mu) C_{t}^{2}\left(m_{t}^{2}, \mu\right) H\left(m_{H}^{2}, \mu\right) \frac{1}{(2 \pi)^{2}} \int d^{2} y_{\perp} e^{i q_{\perp} \cdot y_{\perp}} \\
& \times \tilde{G}_{g / A}^{\mu v}\left(x_{A}, y_{\perp}, S_{A} ; \zeta_{A}, \mu\right) \tilde{G}_{g / B \mu v}\left(x_{B}, y_{\perp}, S_{B} ; \zeta_{B}, \mu\right)+\mathscr{O}\left(q_{T} / m_{H}\right)
\end{aligned}
$$

\section{Evolution and refactorization of gluon TMDPDFs}

The evolution kernel that we derive below implements the evolution of the TMDs with respect to the two scales that they depend on: the renormalization scale $\mu$ and the rapidity scale $\zeta$. 
On one hand, the evolution in terms of the renormalization scale $\mu$ is governed by the anomalous dimension:

$$
\frac{d}{d \ln \mu} \ln \tilde{G}_{g / A}^{[p o l]}\left(x_{A}, b_{\perp}, S_{A} ; \zeta_{A}, \mu\right) \equiv \gamma_{G}\left(\alpha_{s}(\mu), \ln \frac{\zeta_{A}}{\mu^{2}}\right)
$$

where

$$
\gamma_{G}\left(\alpha_{s}(\mu), \ln \frac{\zeta_{A}}{\mu^{2}}\right)=-\Gamma_{\text {cusp }}^{A}\left(\alpha_{s}(\mu)\right) \ln \frac{\zeta_{A}}{\mu^{2}}-\gamma^{n c}\left(\alpha_{s}(\mu)\right)
$$

and $\tilde{G}_{g / A}^{[p o l]}$ represents any of the eight leading-twist (un)polarized gluon TMDPDFs in coordinate space. The coefficients of the perturbative expansions of $\Gamma_{\text {cusp }}$ and $\gamma^{V}$ are known up to three loops.

On the other hand, the evolution equation in $\zeta$ is given by

$$
\frac{d}{d \ln \zeta_{A}} \ln \tilde{G}_{g / A}^{[p o l]}\left(x_{A}, b_{\perp}, S_{A} ; \zeta_{A}, \mu\right)=-D_{g}\left(b_{T} ; \mu\right) .
$$

At small $b_{T}$ the $D_{g}$ term can be calculated perturbatively, but at large $b_{T}$ it has to be modelled and extracted from experimental data. Notice that both the anomalous dimension $\gamma_{G}$ and the $D_{g}$ term drive the evolution of all (un)polarized gluon TMDs.

Given the currently known perturbative ingredients, and regardless of how the non-perturbative tail of the $D_{g}$ term is modelled, we can perform the evolution of all leading-twist gluon TMDPDFs consistently up to NNLL:

$$
\tilde{G}_{g / A}^{[p o l]}\left(x_{A}, b_{\perp}, S_{A} ; \zeta_{A, f}, \mu_{f}\right)=\tilde{G}_{g / A}^{[p o l]}\left(x_{A}, b_{\perp}, S_{A} ; \zeta_{A, i}, \mu_{i}\right) \tilde{R}^{g}\left(b_{T} ; \zeta_{A, i}, \mu_{i}, \zeta_{A, f}, \mu_{f}\right),
$$

where the evolution kernel $\tilde{R}^{g}$ is

$$
\tilde{R}^{g}\left(b_{T} ; \zeta_{A, i}, \mu_{i}, \zeta_{A, f}, \mu_{f}\right)=\exp \left\{\int_{\mu_{i}}^{\mu_{f}} \frac{d \bar{\mu}}{\bar{\mu}} \gamma_{G}\left(\alpha_{s}(\bar{\mu}), \ln \frac{\zeta_{A, f}}{\bar{\mu}^{2}}\right)\right\}\left(\frac{\zeta_{A, f}}{\zeta_{A, i}}\right)^{-D_{g}\left(b_{T} ; \mu_{i}\right)} .
$$

When the transverse momentum is perturbative, the TMDPDFs can be expressed, by performing an operator product expansion (OPE), in terms of collinear functions, integrating out the large transverse momentum by means of Wilson coefficients. This OPE can be written in general, for $b_{T} \ll \Lambda_{\mathrm{QCD}}^{-1}$, as

$$
\tilde{F}_{g / A}\left(x_{A}, b_{T} ; \zeta_{A}, \mu\right)=\sum_{j=q, \bar{q}, g} \tilde{C}_{g / j}\left(x_{A}, b_{T} ; \zeta_{A}, \mu\right) \otimes f_{j / A}\left(x_{A} ; \mu\right)+\mathscr{O}\left(b_{T} \Lambda_{\mathrm{QCD}}\right),
$$

where $\tilde{F}_{g / A}$ represents any of the leading-twist (un)polarized (renormalized) gluon TMDPDFs, and $f_{j / A}$ the corresponding (renormalized) collinear quark/gluon distributions. The coefficients $\tilde{C}_{g / j}$ are different for each case. The convolution refers to momentum fraction $x$ for TMDPDFs that are matched onto twist- 2 collinear functions (like the unpolarized distribution $f_{1}^{g}$ ), while in the case of TMDPDFs that are matched onto twist-3 functions (like the gluon Sivers function $f_{1 T}^{\perp g}$ ) it would represent a two-dimensional convolution in the two momentum fractions of the collinear function. The natural scale for the coefficients $\tilde{C}_{g / j}$ is $\mu \sim 1 / b_{T} \sim q_{T}$, which is the large scale that we integrate out through the OPE. 
Performing the resummation of large logarithms in impact parameter space, the resummed TMDPDF is written as:

$$
\begin{aligned}
\tilde{F}_{g / A}^{\text {Pert }}\left(x_{A}, b_{T} ; \zeta_{A}, \mu\right) & =\exp \left\{\int_{\mu_{0}}^{\mu} \frac{d \bar{\mu}}{\bar{\mu}} \gamma_{G}\left(\alpha_{s}(\bar{\mu}), \ln \frac{\zeta_{A}}{\bar{\mu}^{2}}\right)\right\}\left(\frac{\zeta_{A}}{\zeta_{0}}\right)^{-D_{g}\left(b_{T} ; \mu_{0}\right)} \\
& \times \sum_{j=q, \bar{q}, g} \tilde{C}_{g / j}\left(x_{A}, b_{T} ; \zeta_{0}, \mu_{0}\right) \otimes f_{j / A}\left(x_{A} ; \mu_{0}\right),
\end{aligned}
$$

where $\zeta_{0} \sim \mu_{b}^{2}$ and $\mu_{0} \sim \mu_{b}$. The superscript Pert signifies that it is only valid in the perturbative region $b_{T}<<1 / \Lambda_{Q C D}$. For large $b_{T}$ we need to model them and extract them from experimental data. To do so, one could implement a smooth cutoff that freezes the perturbative contribution slowly as $b_{T}$ gets larger:

$$
\tilde{F}_{g / A}\left(x_{A}, b_{T} ; \zeta_{A}, \mu\right)=\tilde{F}_{g / A}^{P e r t}\left(x_{A}, \hat{b}_{T} ; \zeta_{A}, \mu\right) \tilde{F}^{N P}\left(x_{A}, b_{T} ; \zeta_{A}\right),
$$

where the cutoff prescription could be, for instance:

$$
\hat{b}_{T}\left(b_{T}\right)=b_{c}\left(1-e^{-\left(b_{T} / b_{c}\right)^{n}}\right)^{1 / n},
$$

with $n$ an integer number and $b_{c}$ the parameter that determines the separation between the perturbative and non-perturbative regions. With this implementation, the perturbative contribution dominates at small $b_{T}$, while it gets frozen as $b_{T}$ increases. The non-perturbative model $\tilde{F}^{N P}$ is constrained to be 1 for $b_{T}=0$, and plays an increasingly important role as $b_{T}$ increases.

\section{Gluon helicity TMDPDF}

The gluon helicity TMDPDF, $g_{1 L}^{g}$, represents the distribution of longitudinally polarized gluons inside a longitudinally polarized hadron. Here we explicitly show that, if properly defined as in Eq. (2.3), then rapidity divergences cancel among the collinear and soft matrix elements. We use dimensional regularization with the $\overline{\mathrm{MS}}$-scheme $\left(\mu^{2} \rightarrow \mu^{2} e^{\gamma_{E}} /(4 \pi)\right)$ for ultra-violet (UV) divergences and the $\Delta$-regulator [4] for IR and rapidity divergences. More details can be found in Ref. [8]

The collinear matrix element for the partonic channel of a gluon splitting into a gluon, at NLO, is

$$
\begin{aligned}
\tilde{J}_{1}^{g / g}= & \frac{\alpha_{s}}{2 \pi}\left[\delta(1-x)\left(\frac{\beta_{0}}{2 \varepsilon_{\mathrm{UV}}}+\frac{2 C_{A}}{\varepsilon_{\mathrm{UV}}} \ln \frac{\Delta}{Q^{2}}\right)\right. \\
& +2 C_{A} \delta(1-x) L_{T} \ln \frac{\Delta}{Q^{2}}-L_{T}\left(P_{\Delta g / \Delta g}-\delta(1-x) \frac{\beta_{0}}{2}\right) \\
& +\ln \frac{\mu^{2}}{\Delta} P_{\Delta g / \Delta g}-2 C_{A}\left[\frac{(1-x)\left(1+x^{2}\right)}{x}-\frac{(1-x)^{3}}{x}\right] \ln (1-x)-2 C_{A}\left(\frac{\ln (1-x)}{1-x}\right)_{+} \\
& \left.+\delta(1-x)\left(-\frac{\pi^{2}}{2} C_{A}+\frac{17}{9} C_{A}-\frac{2}{9} n_{f}\right)\right] .
\end{aligned}
$$


The mixed divergences in the result above $\left(\frac{1}{\varepsilon_{\mathrm{UV}}} \ln \Delta\right)$ are rapidity divergences, which are eliminated by subtracting half of the soft function (subtracting and not adding because we need to take care of the double counting of the soft region), which at NLO is:

$$
\tilde{S}_{1}=\frac{\alpha_{s} C_{A}}{2 \pi}\left[-\frac{2}{\varepsilon_{\mathrm{UV}}^{2}}+\frac{2}{\varepsilon_{\mathrm{UV}}} \ln \frac{\Delta^{2}}{\mu^{2} Q^{2}}+L_{T}^{2}+2 L_{T} \ln \frac{\Delta^{2}}{\mu^{2} Q^{2}}+\frac{\pi^{2}}{6}\right] .
$$

The final result for gluon helicity TMDPDF at NLO is then

$$
\begin{aligned}
\tilde{g}_{1 L}^{g / g} & =\frac{\alpha_{s}}{2 \pi}\left\{\delta(1-x)\left[\frac{C_{A}}{\varepsilon_{\mathrm{UV}}^{2}}+\frac{1}{\varepsilon_{\mathrm{UV}}}\left(\frac{\beta_{0}}{2}+C_{A} \ln \frac{\mu^{2}}{\zeta}\right)\right]-L_{T}\left[\mathscr{P}_{\Delta g / \Delta g}-\frac{\beta_{0}}{2} \delta(1-x)\right]\right. \\
& +C_{A} \delta(1-x)\left(-\frac{1}{2} L_{T}^{2}+L_{T} \ln \frac{\mu^{2}}{\zeta}-\frac{\pi^{2}}{12}\right) \delta(1-x)+\mathscr{P}_{\Delta g / \Delta g} \ln \frac{\mu^{2}}{\Delta}+\delta(1-x)\left[\frac{17}{9} C_{A}-\frac{2}{9} n_{f}\right] \\
& \left.-2 C_{A}\left[\frac{(1-x)\left(1+x^{2}\right)}{x}-\frac{(1-x)^{3}}{x}\right] \ln (1-x)-2 C_{A}\left(\frac{\ln (1-x)}{1-x}\right)_{+}-C_{A} \frac{\pi^{2}}{2} \delta(1-x)\right\} .
\end{aligned}
$$

As anticipated, in this result all rapidity divergences have disappeared. The only $\Delta$-dependence, in the second line, parametrizes the infra-red long-distance physics.

Below we illustrate the effect of QCD evolution on gluon helicity TMDPDF, which is implemented as

$$
\begin{aligned}
\tilde{g}_{1 L}^{g}\left(x_{A}, b_{T} ; Q^{2}, Q\right) & =\exp \left\{\int_{\mu_{0}}^{Q} \frac{d \bar{\mu}}{\bar{\mu}} \gamma_{G}\left(\alpha_{s}(\bar{\mu}), \ln \frac{Q^{2}}{\bar{\mu}^{2}}\right)\right\}\left(\frac{Q^{2}}{\zeta_{0}}\right)^{-D_{g}\left(\hat{b}_{T} ; \mu_{0}\right)} \\
& \times \sum_{j=q, \bar{q}, g} \int_{x_{A}}^{1} \frac{d \bar{x}}{\bar{x}} \tilde{C}_{g / j}^{g}\left(\bar{x}, \hat{b}_{T} ; \zeta_{0}, \mu_{0}\right) g_{j / A}\left(x_{A} / \bar{x} ; \mu_{0}\right) \tilde{F}_{j / A}^{N P}\left(x_{A}, b_{T} ; Q\right) .
\end{aligned}
$$

The Wilson coefficients $\tilde{C}_{g / j}^{g}$ are calculated at NLO, for the first time, in Ref. [8]. Here we choose $\zeta_{0} \sim \mu_{0}^{2} \sim \mu_{b}^{2}$, and in order to separate the perturbative and non-perturbative regions we implement the $\hat{b}_{T}$ prescription

$$
\hat{b}_{T}\left(b_{T}\right)=b_{c}\left(1-e^{-\left(b_{T} / b_{c}\right)^{2}}\right)^{1 / 2}, \quad b_{c}=1.5 \mathrm{GeV}^{-1}
$$

with a simple non-perturbative model

$$
\tilde{F}_{j / A}^{N P}\left(x_{A}, b_{T} ; Q\right)=\exp \left[-b_{T}^{2}\left(\lambda_{g}+\lambda_{Q} \ln \left(Q^{2} / Q_{0}^{2}\right)\right)\right], \quad Q_{0}=1 \mathrm{GeV} .
$$

The parameters $\lambda_{g}$ and $\lambda_{Q}$ have never been extracted from experimental data. However $\lambda_{Q}$ is the same among all (un)polarized gluon TMDPDFs, because it parametrizes the scale-dependent part of the non-perturbative model, which is related to the large- $b_{T}$ tail of the universal $D_{g}$ function.

Fig. 1(a) shows the gluon helicity TMDPDF at $Q=20 \mathrm{GeV}$, with some particular choice of the non-perturbative parameters and collinear gluon and quark helicity PDFs obtained from Ref. [16]. It is clear that the theoretical uncertainty gets reduced as we increase the resummation accuracy. In Fig. 1(b) shows the effect of the QCD evolution on gluon helicity TMDPDF for a different choice of non-perturbative parameters. As can be seen, the larger the scale, the wider is the distribution. 


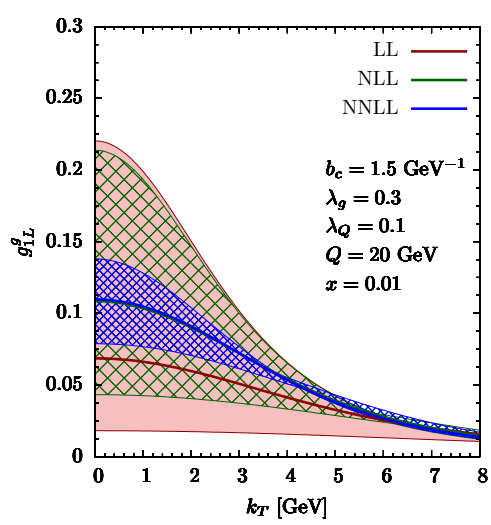

(a)

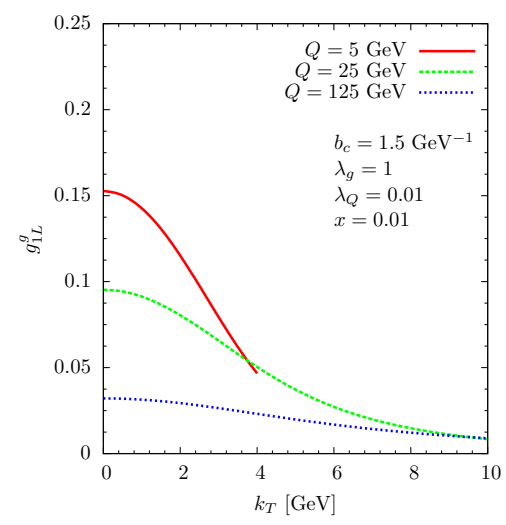

(b)

Figure 1: (a): The gluon helicity TMDPDF $g_{1 L}^{g}$ at $Q=20 \mathrm{GeV}, x=0.01$ and with the non-perturbative parameters chosen to be $\lambda_{g}=0.3$ and $\lambda_{Q}=0.1$. The bands come from varying independently both the resummation scale $\mu_{0}$ and the rapidity scale $\zeta_{0}$ by a factor of 2 around their default value, and taking the maximum variation. (b): The gluon helicity TMDPDF $g_{1 L}^{g}$ at $x=0.01$ for different values of the evolution scale and the non-perturbative parameters, at $\sqrt{s}=8 \mathrm{TeV}$. All curves are given at NNLL accuracy.

\section{Gluon TMDPDFs in an unpolarized hadron}

There are two gluon distributions that contribute at leading-twist in the case of an unpolarized hadron: the unpolarized $\left(f_{1}^{g}\right)$ and the linearly polarized $\left(h_{1}^{\perp g}\right)$ ones. The latter was introduced in [17] and implemented for the first time in the resummation of gluon-gluon fusion process in impact parameter space in $[18,19]$. As described in the previous section for the gluon helicity TMDPDF, both $f_{1}^{g}$ and $h_{1}^{\perp g}$ in the small $b_{T}$ region can be factorized in terms of collinear functions, which in this case are just the unpolarized collinear gluon/quark PDFs:

$$
\begin{aligned}
\tilde{f}_{1}^{g / A}\left(x_{A}, b_{T} ; Q^{2}, Q\right) & =\exp \left\{\int_{\mu_{0}}^{Q} \frac{d \bar{\mu}}{\bar{\mu}} \gamma_{G}\left(\alpha_{s}(\bar{\mu}), \ln \frac{Q^{2}}{\bar{\mu}^{2}}\right)\right\}\left(\frac{Q^{2}}{\zeta_{0}}\right)^{-D_{g}\left(b_{T} ; \mu_{0}\right)} \\
& \times \sum_{j=q, \bar{q}, g} \int_{x_{A}}^{1} \frac{d \bar{x}}{\bar{x}} \tilde{C}_{g / j}^{f}\left(\bar{x}, b_{T} ; \zeta_{0}, \mu_{0}\right) f_{j / A}\left(x_{A} / \bar{x} ; \mu_{0}\right)+\mathscr{O}\left(b_{T} \Lambda_{\mathrm{QCD}}\right), \\
\tilde{h}_{1}^{\perp g / A(2)}\left(x_{A}, b_{T} ; Q^{2}, Q\right) & =\exp \left\{\int_{\mu_{0}}^{Q} \frac{d \bar{\mu}}{\bar{\mu}} \gamma_{G}\left(\alpha_{s}(\bar{\mu}), \ln \frac{Q^{2}}{\bar{\mu}^{2}}\right)\right\}\left(\frac{Q^{2} b_{T}^{2}}{4 e^{-2 \gamma_{E}}}\right)^{-D_{g}\left(b_{T} ; \mu_{0}\right)} \\
& \times \sum_{j=q, \bar{q}, g} \int_{x_{A}}^{1} \frac{d \bar{x}}{\bar{x}} \tilde{C}_{g / j}^{h}\left(\bar{x}, b_{T} ; \zeta_{0}, \mu_{0}\right) f_{j / A}\left(x_{A} / \bar{x} ; \mu_{0}\right)+\mathscr{O}\left(b_{T} \Lambda_{\mathrm{QCD}}\right) .
\end{aligned}
$$

The perturbative coefficients $\tilde{C}_{g / j}^{f, h}$ are given in Ref. [8].

The contribution of unpolarized and/or linearly polarized gluon distributions in unpolarized hadron-hadron collisions depends on the process under study and has been discussed in several works $[10,12,13,14,15]$. In this work we focus on the production of Higgs boson and $C$-even pseudoscalar bottonium state $\eta_{b}$ [20], since for the production of $P$-wave quarkonium states (like $\left.\chi_{b 0}\right)$ there are arguments that suggest a breaking of the factorization [21]. In the considered cases, Higgs boson and $\eta_{b}$ production, both unpolarized and linearly polarized distributions play a role, 

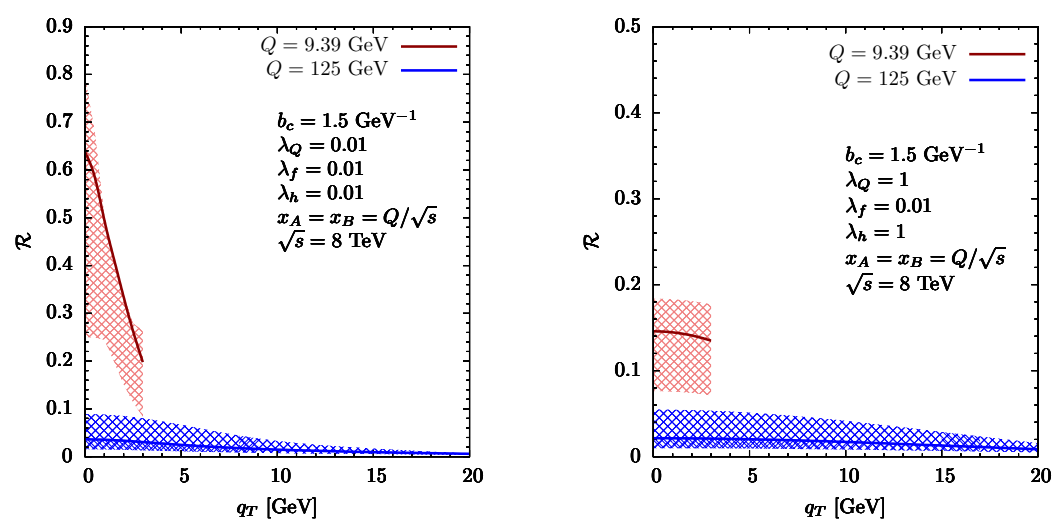

Figure 2: Ratio $\mathscr{R}$ for different values of the non-perturbative parameters $\lambda_{f(h)}$ and $\lambda_{Q}$, at the relevant scales for Higgs boson and $\eta_{b}$ production. The curves are calculated at NNLL accuracy and for $\sqrt{s}=8 \mathrm{TeV}$. The bands come from varying independently both the resummation scale $\mu_{0}$ and the rapidity scale $\zeta_{0}$ by a factor of 2 around their default value, and taking the maximum variation. Notice the differences in scales on the vertical axes.

and thus one can investigate their relative contribution to the cross-section. We use our results to quantify the contribution of linearly polarized gluons, considering the following ratio:

$$
\mathscr{R}\left(x_{A}, x_{B}, q_{T} ; Q\right)=\frac{\int d^{2} b_{T} e^{-i q_{T} \cdot b_{T}} \tilde{h}_{1}^{\perp g / A(2)}\left(x_{A}, b_{T} ; Q^{2}, Q\right) \tilde{h}_{1}^{\perp g / B(2)}\left(x_{B}, b_{T} ; Q^{2}, Q\right)}{\int d^{2} b_{T} e^{-i q_{T} \cdot b_{T}} \tilde{f}_{1}^{g / A}\left(x_{A}, b_{T} ; Q^{2}, Q\right) \tilde{f}_{1}^{g / B}\left(x_{B}, b_{T} ; Q^{2}, Q\right)},
$$

where the numerator and denominator are the two terms in the factorized cross section which determine the relative contributions from linearly polarized and unpolarized gluons, for both Higgs boson and $C$-even pseudoscalar bottonium production. In order to compute this quantity we choose $\zeta_{0} \sim \mu_{0}^{2} \sim \mu_{b}^{2}$ and use the $\hat{b}_{T}$ prescription to separate the perturbative from non-perturbative contributions as in Eq. (4.5). The latter will be parametrized as:

$$
\begin{array}{ll}
\tilde{F}_{j / A}^{f, N P}\left(x_{A}, b_{T} ; Q\right)=\exp \left[-b_{T}^{2}\left(\lambda_{f}+\lambda_{Q} \ln \left(Q^{2} / Q_{0}^{2}\right)\right)\right], & Q_{0}=1 \mathrm{GeV}, \\
\tilde{F}_{j / A}^{h, N P}\left(x_{A}, b_{T} ; Q\right)=\exp \left[-b_{T}^{2}\left(\lambda_{h}+\lambda_{Q} \ln \left(Q^{2} / Q_{0}^{2}\right)\right)\right], & Q_{0}=1 \mathrm{GeV} .
\end{array}
$$

Notice that the parameter $\lambda_{Q}$ is the same for both functions, since the evolution is universal among all (un)polarized TMDPDFs, that is, their scale-dependence is the same. Having precise estimates for this ratio will help us predict the measurability of both unpolarized and linearly polarized gluon distributions in a given process (or scale), which is the final goal.

In Fig. 2 we show our results for the ratio $\mathscr{R}$ at the relevant scales for the transverse momentum distributions of Higgs boson and $\eta_{b}$, all at NNLL accuracy, and for different values of the nonperturbative parameters. Comparing our results to the ones presented in [15], we have included the contribution of quark PDFs to the collinear expansion of gluon TMDPDFs (through $\tilde{C}_{g / q}^{f, h}$ in Eq. (5.1)), performed the resummation consistently at NNLL accuracy and produced consistent uncertainty bands to address the theoretical error. The outcome of the numerical study is clear: the lower the scale the more contribution we have from linearly polarized gluons, although this contribution depends on the value of the non-perturbative parameters, which will have to be fixed by fitting experimental data. 


\section{Summary}

We have derived the factorization theorem for the Higgs boson transverse momentum distribution in hadron-hadron collisions with general polarizations, $A\left(P, S_{A}\right)+B\left(\bar{P}, S_{B}\right) \rightarrow H\left(m_{H}, q_{T}\right)+X$, using the effective field theory methodology. By doing so, we have provided the proper definition of all the leading-twist (un)polarized gluon TMDPDFs, combining adequately the relevant collinear and (part of) soft matrix elements, in order to cancel the spurious rapidity divergences.

The evolution of all leading-twist (un)polarized gluon TMDPDFs is universal, and the currently known perturbative ingredients allow the resummation of large logarithms contained in this evolution kernel up to NNLL accuracy.

We have obtained, for the first time, the NLO Wilson coefficient for the gluon helicity TMD$\operatorname{PDF} g_{1 L}^{g}$, which will allow more accurate phenomenological studies of this quantity in the future, e.g., at RHIC, AFTER @LHC or EIC. We have also obtained the OPE Wilson coefficients for $f_{1}^{g}$ and $h_{1}^{\perp g}$ in the framework presented in this paper. Using these results, we have performed a numerical study of the contribution of linearly polarized gluons for the productions of $\eta_{b}$ and Higgs boson in unpolarized hadron-hadron collisions. The major conclusion is that the larger the relevant hard scale is, the less sensitive is the observable to their non-perturbative contribution, and therefor harder to extract.

\section{References}

[1] J. C. Collins and D. E. Soper, Nucl. Phys. B 194, 445 (1982).

[2] J. C. Collins and D. E. Soper, Nucl. Phys. B 193, 381 (1981) [Nucl. Phys. B 213, 545 (1983)].

[3] J. C. Collins, Foundations of perturbative QCD (Cambridge University Press, Cambridge, 2011).

[4] M. G. Echevarria, A. Idilbi and I. Scimemi, JHEP 1207, 002 (2012) [arXiv:1111.4996 [hep-ph]].

[5] M. G. Echevarría, A. Idilbi and I. Scimemi, Phys. Lett. B 726, 795 (2013) [arXiv:1211.1947 [hep-ph]].

[6] M. G. Echevarria, I. Scimemi and A. Vladimirov, arXiv:1509.06392 [hep-ph].

[7] M. G. Echevarria, I. Scimemi and A. Vladimirov, arXiv:1511.05590 [hep-ph].

[8] M. G. Echevarria, T. Kasemets, P. J. Mulders and C. Pisano, JHEP 1507, 158 (2015) [arXiv:1502.05354 [hep-ph]].

[9] M. G. Echevarria, A. Idilbi and I. Scimemi, Phys. Rev. D 90, no. 1, 014003 (2014) [arXiv:1402.0869 [hep-ph]].

[10] D. Boer, W. J. den Dunnen, C. Pisano, M. Schlegel and W. Vogelsang, Phys. Rev. Lett. 108, 032002 (2012) [arXiv:1109.1444 [hep-ph]].

[11] D. Boer and C. Pisano, Phys. Rev. D 86, 094007 (2012) [arXiv:1208.3642 [hep-ph]].

[12] D. Boer, W. J. den Dunnen, C. Pisano and M. Schlegel, Phys. Rev. Lett. 111, no. 3, 032002 (2013) [arXiv:1304.2654 [hep-ph]].

[13] C. Pisano, D. Boer, S. J. Brodsky, M. G. A. Buffing and P. J. Mulders, JHEP 1310, 024 (2013) [arXiv:1307.3417 [hep-ph]]. 
[14] W. J. den Dunnen, J. P. Lansberg, C. Pisano and M. Schlegel, Phys. Rev. Lett. 112, 212001 (2014) [arXiv:1401.7611 [hep-ph]].

[15] D. Boer and W. J. den Dunnen, Nucl. Phys. B 886, 421 (2014) [arXiv:1404.6753 [hep-ph]].

[16] D. de Florian, R. Sassot, M. Stratmann and W. Vogelsang, Phys. Rev. Lett. 113, no. 1, 012001 (2014) [arXiv:1404.4293 [hep-ph]].

[17] P. J. Mulders and J. Rodrigues, Phys. Rev. D 63, 094021 (2001) [hep-ph/0009343].

[18] S. Catani and M. Grazzini, Nucl. Phys. B 845, 297 (2011) [arXiv:1011.3918 [hep-ph]].

[19] S. Catani and M. Grazzini, Eur. Phys. J. C 72, 2013 (2012) [Eur. Phys. J. C 72, 2132 (2012)] [arXiv:1106.4652 [hep-ph]].

[20] J. P. Ma, J. X. Wang and S. Zhao, Phys. Rev. D 88, no. 1, 014027 (2013) [arXiv:1211.7144 [hep-ph]].

[21] J. P. Ma, J. X. Wang and S. Zhao, Phys. Lett. B 737, 103 (2014) [arXiv:1405.3373 [hep-ph]]. 